\title{
Use Cases of Real-Time Locating Systems for Factory Planning and Production Monitoring
}

\author{
Alexander Mütze ${ }^{\mathrm{a}, *}$, Lennart Hingst ${ }^{\mathrm{a}}$, Niklas Rochow ${ }^{\mathrm{a}}$, Timo Miebach ${ }^{\mathrm{a}}$, Peter Nyhuis ${ }^{\mathrm{a}}$ \\ ${ }^{a}$ Institute of Production Systems and Logistics, Leibniz University Hannover, An der Universität 2, 30823 Garbsen, Germany
}

\begin{abstract}
Production companies operate in an increasingly dynamic and unpredictable market environment. The ever shorter innovation cycles and the associated decreasing product life cycles require production systems and processes to change continuously. Furthermore, rising complexity in market enfactories through product variety leads to an increased coordination effort in factory operations. To stay competitive, decision making in factory planning as well as production planning and control (PPC) must be done target-oriented and in the shortest time possible. A fundamental basis in this respect is a reliable and consistent database, needed for the overall and holistic improvement of the logistics performance of a company's internal supply chain. However, the collection and preparation of data is associated with a high expenditure of time and thus represents a great challenge. Additionally, required data in practice is often inhomogeneous or inconsistent, which makes it difficult to track ongoing processes precisely. Due to the continuous availability of exact positions at any time, Real-Time Locating Systems (RTLS) offer the potential to provide movement data of essential elements of the production system such as material supply, resources and workforce with comparatively little effort. To demonstrate the resulting potentials for factory planning and PPC, use cases for RTLS have been identified and implemented in the Learning Factory of the Institute for Production Systems and Logistics (IFA). After a short introduction of the IFA Learning Factory and the principle of Real-Time Locating Systems, the use cases in factory planning, factory operation and production monitoring are described. Having summarized the achieved benefits provided for the training participants in the IFA Learning Factory a summarizing conclusion is given, underlining the great possibilities of real-time localization in production environments and providing an outlook on further research activities.
\end{abstract}

(C) 2021 The Authors. This is an open access article.

Peer-review statement: Peer-review under responsibility of the scientific committee of the 11th Conference on Learning Factories 2021

Keywords: Real-Time Locating System; RTLS; Factory Planning; Factory Operation; PPC; Monitoring; Learning Factory

\section{Introduction}

Manufacturing companies face the challenge of continuously improving and adapting their production systems to changing conditions in order to remain competitive [1]. Especially due to shortened product life cycles, the ability to steadily adapt to changing conditions within factory planning and operation is becoming increasingly important [1,2]. For this reason, it is essential to improve transparency regarding the processes within the factory, to implement useful technologies for collecting (real-time) data and to use the provided information effectively and in an appropriate way [3,4]. This way, previous barriers in achieving production logistics objectives, such as a high schedule reliability and low delivery times towards the customer, can be overcome.

One way to face this challenge is the real-time localization of production resources, materials and orders with so-called Real-time Locating Systems (RTLS) [5,6]. In order to explore its possibilities and to derive possible use cases to support the holistic production system design and its operation, such a system was implemented in the IFA Learning Factory [7], where trainings for industry and students are conducted in the field of production organization. In the following, this paper presents the basics of holistic production system design and RTLS, a short overview on the IFA Learning Factory and the derivation of use cases for RTLS application.

\footnotetext{
* Corresponding author. Tel.: +49-511-762-18183

E-mail address: muetze@ifa.uni-hannover.de
} 


\section{Fundamentals towards a holistic production system design}

The holistic design of production systems ensures value-adding processes as well as material and information flows in line with the company's overall objectives, avoiding isolated solutions [8]. With regard to the planning horizon, a distinction between short-, medium- and long-term adjustments can be made. Long-term adjustments refer to the investment planning and determination of the capacities of a factory as part of factory planning. Medium-term adjustments involve the reorganization of work processes to deliver the required output, and shortterm adjustments are made as part of production planning and control (PPC) to ensure optimum use of operating resources [9]. The adjustments can be consequently broken down to factory planning and factory operation. Factory planning represents the pre-planned value creation for the production of industrial goods, while factory operation is dedicated to the realization of competitive value creation of industrial goods [10]. The tasks of factory planning include the personnel, technical-organizational design of the processes as well as their elements and structures in conjunction with the organizational structure [11]. In contrast, factory operation runs, manages and monitors the processes in the factory in order to ensure the company's objectives are met through the interaction of people, technology and organization as well as cooperation within and outside the factory [10]. To stay competitive in a volatile, uncertain and turbulent market environment, decision making in factory planning as well as PPC, representing factory operation, is continuously required and must be done target-oriented and in the shortest time possible. For this purpose, a homogeneous and consistent database and an efficient production monitoring is needed to reduce the effort required for data collection as part of the continuously occurring tasks. RTLS therefore can be a way to create a reliable database as a basis for synchronized planning activities. This ensures that factory planning provides design solutions and potentials that can be used by factory operation for different target alignments [10].

\section{IFA Learning Factory: An Interactive Training Environment}

The IFA Learning Factory is a modular training environment in which participants from both university and industry can experience the latest research findings in the field of production system design. The covered training topics range from the Factory Planning Toolbox to Lean Production and Production Planning and Control. Subjects relating to workplace design and ergonomics are also addressed. The diversity of the offered training courses as well as the high adaptability to the specific requirements of the training participants is ensured by a highly flexible production environment in which technical support systems can be added in a modular way. In addition the production area itself can be arranged freely. To ensure continuity among the training courses, the product in all training courses of the IFA Learning Factory is a metal model helicopter. For this, depending on the training, either the components are manufactured in a job shop production or the manufactured components are assembled in a finishing assembly line. [3,7,12,13]

In line with developments in the field of production system design, the IFA Learning Factory is continuously evolving in terms of both content and technology. In recent years, technologies such as RFID, electronic shelf labels (ESL), smart glasses and the RTLS, which is in the focus of this article, have been implemented. Current development activities include the implementation of automated-guided-vehicles (AGVs), laser labelling devices and a 3D laser scanner for measuring production areas providing data for virtual reality applications. Through exemplary use in simulation games, the training participants are shown various possibilities supporting decisionmaking in production logistics. The round-based simulation games are the practical part in training courses where participants recreate a use case by assuming a specific role [13]. After each round, the training participants have the opportunity to implement changes. After decision-making, participants can experience consequences and errors in a practical way. The use of an RTLS offers the possibility to highlight this transparently already during the game round and enables a faster response. The corresponding depth of data after another game round has been completed allows training participants to make more informed decisions. Subsequently, it gives the participants the opportunity to reflect whether the actions taken have brought the desired success, thus increasing the problemsolving competence of the participants and allowing them to develop a deeper understanding of the operations [7].

\section{Real-Time Locating System in the IFA Learning Factory}

Existing IT system infrastructure is based on discrete data acquisition using static feedback points. The systems are structured hierarchically into plant, section, and workstation levels. Data tracking on factory and section level does not pose a challenge. However, the static feedback system usually excludes the workstation level. Here, the time between two booking points is regarded as a black box resulting in a lack of information (e.g. idle times, spatial data). Furthermore, the administration and booking effort increases significantly. The feasibility and implementation limits usually lie at the workstation level. Therefore, the advantages of RTLS compared to conven- 

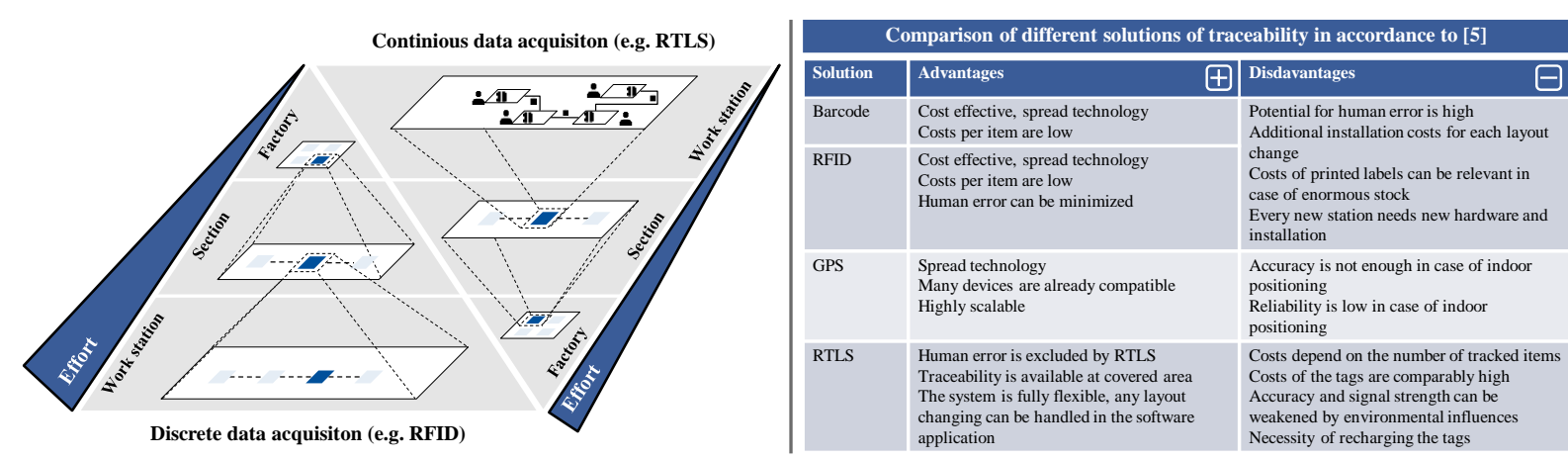

Fig. 1. Top-down and bottom-up data acquisition of the different material flow levels and comparison of different traceability solutions.

tional traceability solutions (e.g. at PDA terminals) include high data consistency resulting from continuous instead of discrete recording of feedback data. The real-time capability and the possibility of using RTLS both in continuous operation and for one-time analyses are accompanied with a higher precision than for example GPS. On the other hand, there are disadvantages such as higher acquisition costs and, depending on the technology used, the need to periodically recharge the tags (see Figure 1).[5,6] Overall, RTLS serves as a suitable technology for closing the black box increasing the information density about running processes.

RTLS consist of the basic components: Tag (applied to the object to be tracked), anchors (reference points in the corners of the catchment area tracing the signals) and server (calculation of the object's position based on the information flow provided by the anchors). RTLS therefore are based on radio frequency communication, often using the ultra-wideband (UWB-) technology $[5,6]$. The localization is based on the principle of triangulation and the use of geometric properties of a triangle [6]. ${ }^{1}$ In the IFA Learning Factory, a RTLS using UWB technology and the Time of Arrival-principle is implemented. The position of the tag is thus determined by the computed distance between the tag and at least 3 anchors. For the most accurate determination, 6 anchors were attached to the ceiling grid, and a server writes the acquired data to an SQL database, providing an open interface for the usage with other systems.

\section{Use-Cases for the effective usage of Real-Time Locating Systems}

The challenges in production system design are based on the fact that the necessary adjustments in the factory are not made once, but have become a permanent task of various specialists and disciplines. These adjustments influence each other. The long-term decisions have an impact on the adjustment possibilities of the medium-term reorganization of work processes. At the same time, the reorganization measures define the scope for short-term adjustments in the field of planning and control [9]. This makes it all the more important to have a reliable and consistent database as the basis for a target-oriented and holistic design of a production system [8]. Due to the continuous availability of exact positions at any time, it is possible to provide movement data for the adjustment tasks at short notice with the help of the RTLS. For this reason, use cases have been collected for factory planning and PPC with a focus on production monitoring in order to demonstrate the resulting potential. One use case each was elaborated in more detail in order to display the implementation in the context of the IFA Learning Factory.

\subsection{Factory Planning}

Long-term adjustments in factory planning include the design of the production processes with the logistics processes and the necessary supporting processes [15]. In this context, four planning cases are distinguished which differ regarding the scope of planning tasks and problems as well as the restrictions [11]. Factory planning is triggered by either changes within factory or changes within the company or in the factory environment [16]. The subsequent factory planning process (FPP) comprises seven successive phases, carried out with the help of methods and tools. Experience has shown that the most important and usually most time-consuming phase in FPP is the determination of the project basis [9]. This is where information required for planning is collected which serve as the planning basis and input for factory planning [11]. Product and process data are analyzed in order to describe reference processes for the newly planned factory in the case of development planning and to determine the weaknesses and potentials of an existing factory in the case of replanning [16]. Since RTLS can only assist in capturing movement data during ongoing operation, the following use cases deal with the latter case. They describe how data can be collected by tracking products, components, employee and equipment.

\footnotetext{
${ }^{1}$ Within the scope of this paper, no more detailed consideration of the technical functioning is given and reference is made to $[5,6,14]$.
} 


\section{Use case: Tracking of products within a factory in real-time.}

In the establishment of the project basis, it is advantageous to first obtain a quick overview of the production processes. One of the most helpful methods for quick orientation is value stream mapping (VSM) which uses standardized symbols to record the entire value stream of a product family with the associated material and information flows [8]. The aim of VSM is to identify ways of achieving flow-oriented production by eliminating waste in inventories, floor space and throughput times of products. VSM only considers one product or product group at a time [15]. For several products or product families, a relatively large number of analyses is required. RTLS can help to reduce the effort for data acquisition by tracking an order or a product continuously in real time within the factory. The data recorded this way enables VSM 4.0 by further processing the recorded data and automatically calculating the necessary key figures as input variables (e.g. throughput time, see section 4.2) [16].

\section{Use case: Real-time material flow analysis for layout planning}

Generally, there is no reference product for the entire factory in the course of factory planning. Instead, material and information flows should also be analyzed from the resource perspective. A factory often produces about three to five product groups with different quantities and for different customers, so that the products usually compete for capacity of the machines. Therefore, visualizing the flows between operational sections can provide vast planning support [16]. One of the main tasks in factory planning is to determine a favorable spatial arrangement of objects to ensure a directed flow of materials. The goal is to minimize spatial distances between objects with a high material flow intensity $[9,15]$. The material flow is established by the sequence of logistical (movement and storage processes) and value-adding (manufacturing processes) processes. Following the system levels of a factory, the material flow can also be divided hierarchically into different levels. The inter-area material flow levels factory and section represent the macro level and are largely determined by the arrangement of buildings as well as the path network. The inner-area material flow level describes the micro level within a section and depends on the arrangement of the individual workstations [15]. With conventional traceability solutions, the real material flow can be approximated via production supply areas or bookings between the individual work steps. However, it will not be possible to capture the exact material, personnel or resource flow in this way. RTLS can offer a solution by continuously recording position data and converting it into movement data. Raw data at workstation level can be aggregated up to the macro levels through clustering in any way. Data processing depends on the desired tabular and graphical format for capturing, displaying and evaluating material flow shown in Figure 2.

Before different kinds of formats of material flows can be generated, data collection and data preparation must be carried out. First, the data generated by the RTLS is transferred in real time to the mentioned SQL database in the IFA Learning Factory (steps 1 and 2). This data is imported from SQL into MS Excel using interface programming (step 3) and is further processed as part of a cluster analysis (step 4). The cluster analysis is only necessary, if material flow relationship plots are to be displayed. They are an aggregated, usually quantitative form of material flow analysis, which is particularly used for the inter-area material flow level [17]. Cluster analysis is performed based on the known positions of each equipment. The movements between the equipment are identified through an analysis of the raw data using a Visual Basic for Applications (VBA) tool by assigning the position data of the tags to the resources. Two sequentially identified resources are forming a source-sink relationship, so that the field at the intersection of the matrix is counted up for every determined relationship. Sankey diagrams are used for the visual representation of material flow matrices. They give an idea of the degree of interconnection and the quantitative relationships of the material flow.

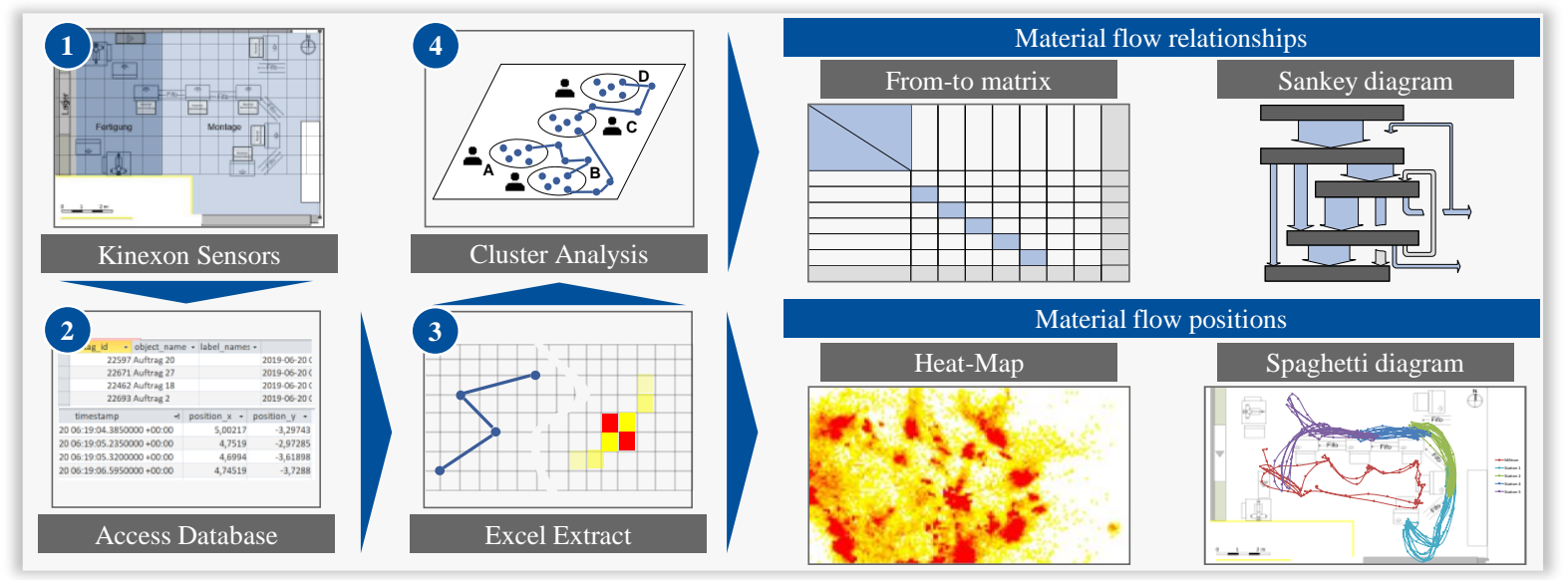

Fig. 2. Procedure for the creation of tabular and graphical formats of material flows in the IFA Learning Factory. 
Representations of material flow positions belong to qualitative material flow analyses that locate the individual movements of materials, people or resources in the layout. In order to ensure transparency, this representation is only suitable for a limited area with a limited number of objects of observation [17]. Therefore, they are particularly used on the inner-area material flow level, in the context of the detailed layout planning of workstations. The RTLS is particularly suitable because continuous data collection is performed. There is no other viable alternative than a manual collection via multimoment sampling. Since only individual movements are visualized in the context of heat maps or spaghetti diagrams, the MS Excel extract requires no further processing. After the data has been reduced in its extent (step 3) by limiting the time period, the time interval and the object of observation (orders), as well as performing an import, the selected position data can be mapped directly on a coordinate system.

\section{Use case: Automatic recording of an existing factory layout}

The visualization of the production processes in combination with real-time data acquisition makes it possible to design a changeable production system. Instead of tracking moving elements such as material, equipment or personnel, the RTLS can thus be used to record factory layouts. Given the need for changeability and transparency of modern production systems, the goal is to automatically record and visualize existing factory layouts. This is done in order to reduce the time-consuming process of recording a factory layout. The live position data required for this is provided by the RTLS. With four small sensors on each workstation, the position data is recorded and collected in an SQL database. The data sets obtained are imported into MS Excel, transferred to the current factory layout and saved as an image file with the help of a VBA tool.

\subsection{Factory Operation and Production Monitoring}

The target-oriented planning and control of the factory requires the continuous monitoring of set parameters as well as of the logistics target achievement [18]. Moreover, in order to avoid wastage in processes, it is important to create a real-time image of production, on which basis decisions can be made in real time, e.g. about processing sequences or transportation jobs. In order to avoid duplications and inconsistencies in data acquisition, it is also necessary to question which technology can be used most sensibly to support factory operation. In the following, use cases for process improvement and avoidance of wastage, as well as for the recording of production feedback points of the production processes in the context of production monitoring, will be presented.

\section{Use case: Tracking of moving objects, fleet control and contact tracing}

Besides the objects to be transported, RTLS can also be used to track the people and resources moving around the factory. This allows a variety of potentials to be leveraged. On the one hand, transparency is increased with regard to the location of equipment and vehicles. Such information could then be used to locate broken-down transport systems, as well as to find e.g. the next available forklift to complete a transportation order.[5] Based on the position of the resources available at any time and the transparency of the actual tasks, it is also possible to significantly improve fleet management. In addition, navigation of e.g. AGVs could be based entirely on RTLS instead of laser sensors or similar. Another possible application, which might be of great importance, especially in the current pandemic situation, is the contact tracing of employees, which is considerably more precise using RTLS than existing tracing apps on smartphones that are based on Bluetooth technology [19].

\section{Use case: Reduction of the efforts to search for orders and materials}

A problem frequently observed in practice is the occurrence of unnecessary processes (wastage) due to the search for materials, orders and products. Thereby, the search efforts can be of various nature, for example, there might be large storage places that are not further subdivided or incorrect bookings leading to a search being conducted at the wrong place. To avoid the resulting (time) wastage, the objects or the load carriers can be equipped with tags so that the exact position is determinable at any time. If the position information is linked to the ME/PPC System and the work schedule, inconsistencies between the expected and the actual location could be detected before productivity losses occur. For more precise identification, especially in places with a large number of objects, electronic-shelf labels can also be used in addition to minimize the required search efforts [12].

\section{Use case: Real-time order tracking and monitoring system requiring little effort to collect feedback data}

The analysis of production feedback data is a significant success lever for efficient and target-oriented factory operation. Thus, as part of a holistic production monitoring, parameters and procedures of PPC can be adjusted and the achievement of production logistic objectives of individual workstations, production areas and the entire factory can be measured [18]. To visualize and analyze the production feedback data the so-called logistic models are well established [20]. These do not only include models that describe the effect of actuating and control variables on the objectives of the object under investigation. Also descriptive models like the throughput element, which subdivides the operation-related throughput time into different time segments giving an overview of the pro- 


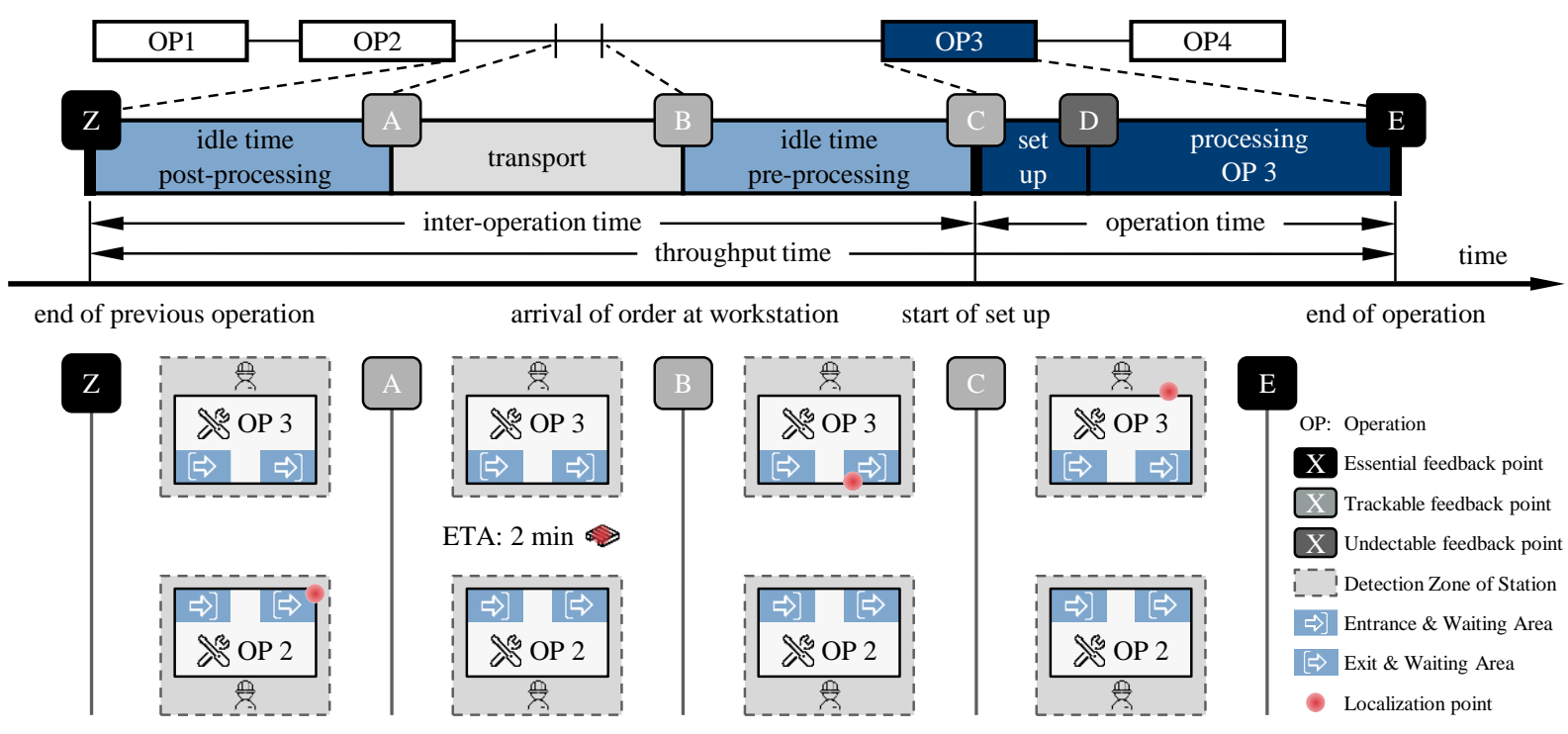

Fig. 3. Throughput Element with feedback points and visualization of corresponding position information of the RTLS (partly based on [12,21]).

-portions of the throughput time and thus of the ratio of operation and inter-operation time, are covered [21]. As shown in Figure 3, the throughput time of an order at a workstation can be subdivided into 5 time intervals, which belong to either the operation time or the inter-operation time. The throughput element thereby follows the logic that the completion of an operation, in Figure 3 marked by feedback point $\mathrm{E}$, is equal to the entry $\mathrm{Z}$ to the following operation. Therefore, it is necessary to actively record 5 feedback points per operation in order to differentiate all shown time segments [3].When using a classic PDA, there is a high effort for all needed bookings of the feedback points. Often this also leads to the fact that bookings are postponed by the employees and are collectively carried out to avoid e.g long walks between the workplace and the terminal. As a result, the MES and/or PPC system does not have an up-to-date image of the production useable as a basis to conduct real-time control decisions.

In contrast, by means of the RTLS it is possible to use the real-time position data to effectively subdivide the throughput time. For this purpose, areas, also called fences, must be defined for the RTLS to make a booking in the ME-/PPC system system when an order (in the form of the connected tag) enters this area for a certain duration. This is illustrated exemplarily by the lower part of Figure 3. It is thus possible to record dedicated feedback points in addition to the anyway provided real-time data by the RTLS without any additional effort for the worker, and thus to differentiate time segments. Consequently, it is possible to obtain an exact picture of the current queue length of the workstation at any time. In addition, booking errors are avoided, which enables the ME-/PPC system to make better, real-time decisions, e.g. to change the sequence at the workstation. Hereby, the achievement of the logistical objectives of the overall system can be influenced in a very short period of time.

Nevertheless, it should also be noted that the possibility of an automatic booking of feedback points by means of RTLS depends on how precisely the position of the objects can be recorded and how large the positional differences of e.g. queuing area and processing area are. In particular, feedback point D can only be recorded in some constellations. It differentiates the setup process from the processing process. Such a differentiation using RTLS is only possible if a significant change in position is noticeable between setup and processing. If e.g. the load carrier or the element carrying the tag remains at the same position, D cannot be derived and it may be necessary to manually book or connect another data providing system in order to log feedback point D.

In summary, RTLS offer many opportunities to improve data quality and quantity of production feedback data and enable a more accurate planning and control of the ongoing production process. However, it has to be noted that already the feedback points $\mathrm{C}$ and $\mathrm{E}$ provide many opportunities for a target-oriented production monitoring and a full record of all feedback points have to be balanced with the necessary effort. An overview of the usage of production feedback data in the IFA Learning Factory was given by SCHÄFERS ET AL. [3].

\section{Benefits of the implementation of RTLS for the conducted trainings in the IFA Learning Factory}

The implementation of RTLS enriches the trainings conducted in a wide range of topics. Thus, the training participants can freely decide which production feedback technology they want to use for the recording of operating data, for example. The options range from manual data acquisition via touch screens, barcode / QR, RFID and 
RTLS. Through the gamified experience in the training game, the participants are able to try out the technologies without fear of failure and build up a competence in the use of the technical systems. The creativity enhancing equipment of the IFA Learning Factory also supports the creativity process, so that new ideas of combining different technologies for different purposes become possible and due to the existing modularity in most cases also realizable. The transfer of professional knowledge in the field of production system design thus merges with the participants' acquisition of competencies in both organizational and digitalization areas.

\section{Conclusion and Outlook}

Within this contribution, use cases from the field of factory planning and operation were used to demonstrate how the application of RTLS can effectively support production system design as a multi-layered and complex challenge. The solutions shown represent potentials that have been revealed through the implementation and study of a RTLS in the IFA Learning Factory and conceived and partially developed with student support. Furthermore, it was also shown what potential lies in a consistently high availability of data in the area of production system design. In the IFA Learning Factory, the main aim of research activities is to demonstrate training participants from industry and students the possibilities offered by digital technologies in the field of production system design. Therefore the focus is on creating transparency and improving production processes as well as the process of factory planning. Student involvement in the context of tutorials, student research projects and thesis is also focused. Thereby, the main purpose is always to show meaningful potentials of digitization and, above all, the practical benefits for production organization avoiding to introduce digital systems for the sake of self-purpose.

Further activities in the IFA Learning Factory are currently focusing the possibility of using AGVs in changing production layouts (combination of presented use cases). For this purpose, the results of automatic layout recording by means of the RTLS are transmitted automatically to the AGV system using a programmed interface. Once the layout is changed, the routing system can be updated with the current positions of the workstation and material supply positions. Consequently, it will be possible to use AGVs even if the production layout is subject to strong, continuous changes. Combined with these development steps, real-time control of production with dynamic routing, i.e. the dynamic assignment of orders to workstations, is also to be supported by AGVs. Central control decisions can thus be implemented quickly and agilely on the shop floor.

\section{References}

[1] D. Mourtzis, Challenges and future perspectives for the life cycle of manufacturing networks in the mass customisation era, Logist. Res., 9 (2016) $1-20$.

[2] G. Schuh, A. Kampker, C. Wesch-Potente, Condition based factory planning, Prod. Eng. Res. Devel., 5 (2011) $89-94$.

[3] P. Schäfers, A. Mütze, P. Nyhuis, Integrated Concept for Acquisition and Utilization of Production Feedback Data to Support Production Planning and Control in the Age of Digitalization, Procedia Manufacturing, 31 (2019) 225-231.

[4] C. Mundt, M. Winter, T. Heuer, M. Hübner, M. Seitz, M. Schmidhuber, J. Maibaum, L. Bank, S. Roth, P. Scherwitz, P. Theumer, PPSReport 2019: Studienergebnisse, 1st ed., TEWISS, Garbsen, 2020.

[5] A. Rácz-Szabó, T. Ruppert, L. Bántay, A. Löcklin, L. Jakab, J. Abonyi, Real-Time Locating System in Production Management, Sensors (Basel, Switzerland), 20 (2020).

[6] J. Slovák, P. Vašek, M. Šimovec, M. Melicher, D. Šišmišová, RTLS tracking of material flow in order to reveal weak spots in production process, $201922^{\text {nd }}$ International Conference on Process Control (PC19), (2019) 234-238.

[7] K.-F. Seitz, P. Nyhuis, Cyber-Physical Production Systems Combined with Logistic Models - A Learning Factory Concept for an Improved Production Planning and Control, Procedia CIRP, 32 (2015) 92-97.

[8] M. Bellgran, K. Säfsten, Production development: Design and operation of production systems, Springer, London, 2010.

[9] G. Pawellek, Ganzheitliche Fabrikplanung: Grundlagen, Vorgehensweise, EDV-Unterstützung, Springer, Berlin, Heidelberg, 2008.

[10] M. Schenk, S. Wirth, E. Müller, Factory Planning Manual: Situation-Driven Production Facility Planning, Springer, Berlin, 2014.

[11] Verein Deutscher Ingenieure, Factory planning - Planning procedures: VDI 5200, 2011.

[12] P. Schäfers, A. Mütze, P. Nyhuis, Digital Production Order Processing Support System Using Real Time Data, in: D. Dimitrov, D. Hagedorn-Hansen, K. von Leipzig (Eds.), International Conference on Competitive Manufacturing (COMA 19), (2019) $28-34$.

[13] M. Quirico, P. Schäfers, P. Nyhuis, Production Controlling In The Age of Digitalization: A Learning Factory Concept For Interactive Education, 2017 IACB, 2017 ICE \& 2017 ICTE Proceedings, (2017) 310-1 - 310-8.

[14] D. Gyulai, A. Pfeiffer, J. Bergmann, Analysis of asset location data to support decisions in production management and control, Procedia CIRP, 88 (2020) 197-202.

[15] C.-G. Grundig, Fabrikplanung: Planungssystematik - Methoden - Anwendungen, 6th ed., Hanser, München, 2018.

[16] H.-P. Wiendahl, J. Reichardt, P. Nyhuis, Handbook Factory Planning and Design, 2015th ed., Springer, Berlin, Heidelberg, 2015.

[17] D. Arnold, K. Furmans, Materialfluss in Logistiksystemen, Springer, Berlin, Heidelberg, 2009.

[18] L. Härtel, P. Nyhuis, Systematic Data Analysis in Production Controlling Systems to Increase Logistics Performance, in: R. Schmitt, G. Schuh (Eds.), Advances in Production Research. WGP 2018. Springer International Publishing, Cham, (2019) 3-13.

[19] H.J. Ho, Z.X. Zhang, Z. Huang, A.H. Aung, W.-Y. Lim, A. Chow, Use of a Real-Time Locating System for Contact Tracing of Health Care Workers During the COVID-19 Pandemic at an Infectious Disease Center in Singapore: Validation Study, Journal of Medical Internet Research, 22 (2020).

[20] P. Nyhuis, H.-P. Wiendahl, Fundamentals of Production Logistics, Springer Berlin Heidelberg, Berlin, Heidelberg, 2009.

[21] W. Heinemeyer, Die Analyse der Fertigungsdurchlaufzeit im Industriebetrieb. Hannover, Techn. Univ., Diss. 1974. 